\title{
Sudden cardiac arrest with pericardial contrast during computed tomography aortogram in a type $A$ aortic dissection patient
}

Hong Kong Med J 2019;25:407.e1-2

https://doi.org/10.12809/hkmj197818

A 69-year-old man with long-standing hypertension and history of pulmonary embolism on warfarin presented to Accident and Emergency Department with sudden upper back pain and transient loss of consciousness. On admission, the patient was in haemodynamic shock with blood pressure 83/57 mm $\mathrm{Hg}$ and pulse 42 beats per minute. Cardiovascular examination revealed radial-radial and radialfemoral delay. Electrocardiogram showed complete heart block and chest radiograph demonstrated

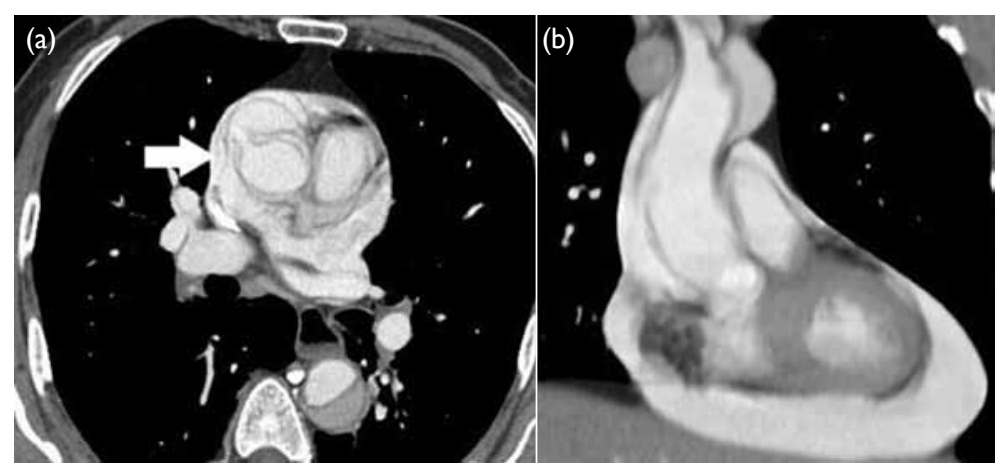

FIG I. Axial (a) and coronal (b) computed tomography aortogram images showing the extension of the dissection flap into pericardial sac. The rupture was seen at the right lateral wall of the ascending aorta (white arrow)

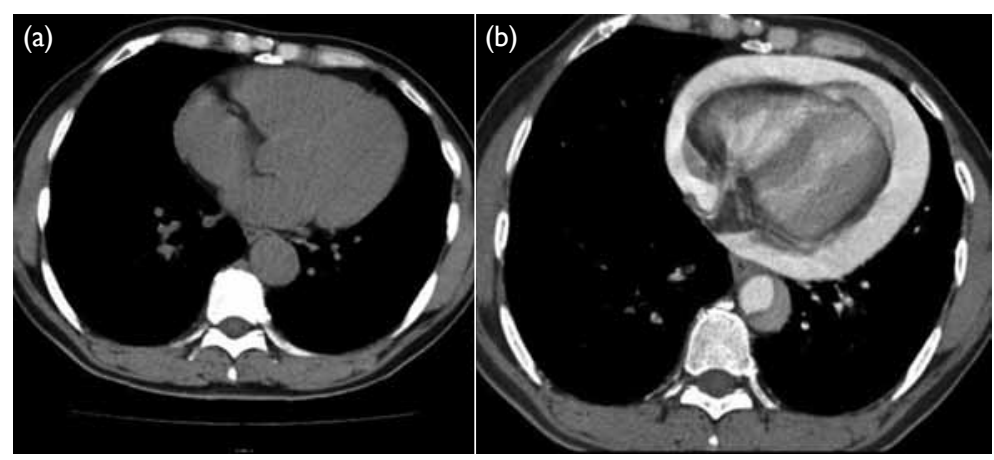

FIG 2. (a) Pre-contrast computed tomography image showing normal pericardium. (b) Computed tomography aortogram image showing significant contrast in the pericardium. A dissection flap can be seen within the descending thoracic aorta. The false lumen was attenuated due to delayed opacification. The right atrium was obliterated, in line with cardiac tamponade a widened mediastinum. An immediate bedside echocardiogram found an intimal flap in the aortic root with evidence of aortic regurgitation and a thin rim of pericardial effusion.

Fluid resuscitation and inotropic support was given and urgent computed tomography (CT) aortogram was arranged to further delineate the extent of involvement of aortic dissection. However, the patient developed sudden cardiac arrest immediately after being given $80 \mathrm{~mL}$ intravenous Omnipaq at a rate of $3 \mathrm{~mL} / \mathrm{s}$ via injector during CT aortogram. A review of CT images showed Stanford type A aortic dissection with an intimal flap extending into the pericardial sac (Fig 1). No obvious pericardial effusion was visible on the pre-contrast $\mathrm{CT}$ images (Fig 2a). However, significant contrast was seen in the pericardial sac on post-contrast CT images (Fig 2b), indicating a sudden rupture with haemopericardium had occurred during the CT scan. Despite prompt bedside pericardiocentesis and cardiopulmonary resuscitation, the patient died.

Aortic dissection is the most common acute emergency condition of the aorta. The mortality rate is high, and rupture is the cause of death in approximately one-third of affected patients. ${ }^{1}$ The pathology is due to a tear in the intimal layer allowing blood to propagate into the media and create a false lumen. In the ascending thoracic aorta, the primary tear is most often within $3 \mathrm{~cm}$ of the aortic cusps. ${ }^{2}$ The false lumen of aorta may rupture due to loss of elastic recoil and increased wall stress with dilatation. Often, the rupture site is close to the initial intimal-medial tear over the right lateral wall where it receives the ejected blood from the left ventricle. ${ }^{2}$ This ends up into the pericardial sac causing haemopericardium and subsequent fatal cardiac tamponade. ${ }^{2}$

Computed tomography aortogram is the firstline modality in the diagnosis of aortic dissection, delineation of its extent of involvement and endorgan ischaemia. In our case, rupture of the aorta into the pericardial sac was evidenced by significant contrast-enhanced haemopericardium on CT aortogram images which was absent in pre-contrast CT images. This could be related to the rapid injection of a large volume bolus of intravenous contrast by power-injector, resulting in a sudden elevation of left ventricular pressure, supported by 
previous study in human subjects demonstrating a significant increase in blood pressure after bolus injection of low osmolarity, non-ionic contrast agent. $^{3}$

The treatment of type A aortic dissection with rupture is immediate surgical repair. ${ }^{4,5}$ Although it was previously considered controversial to perform pericardiocentesis as there is a risk of worsening the leak, recent evidence suggests that controlled pericardiocentesis may reduce haemodynamic instability in critical cardiac tamponade to allow sufficient time for urgent operative repair. ${ }^{5}$

\section{Author contributions}

WM Yu and FH Ng are responsible for the concept of study, acquisition and analysis of data, and drafting of the article. All authors are responsible for critical revision for important intellectual content. All authors had full access to the data, contributed to the study, approved the final version for publication, and take responsibility for its accuracy and integrity.

\section{Conflicts of interest}

All authors have disclosed no conflicts of interest.

\section{Funding/support}

This research received no specific grant from any funding agency in the public, commercial, or not-for-profit sectors.

\section{Ethics approval}

The present study was reviewed and approved by the Kowloon Central Cluster/Kowloon East Cluster Research Ethics
Committee (KCC/KEC-2019-0179). Because the concerned patient was deceased, the requirement for consent was waived by the ethics board.

${ }^{1} \mathrm{WM} \mathrm{Yu}^{*}$, MB, ChB, FRCR

${ }^{2} \mathrm{FH} \mathrm{Ng}, \mathrm{MB}, \mathrm{ChB}, \mathrm{FRCR}$

${ }^{1}$ WL Wong, MB, ChB, FRCR

${ }^{1}$ Department of Radiology, United Christian Hospital, Kwun Tong, Hong Kong

2 Department of Radiology, Caritas Medical Centre, Shamshuipo, Hong Kong

* Corresponding author: wenmingyu@hotmail.com

\section{References}

1. Mehta RH, Suzuki T, Hagan PG, et al. Predicting death in patients with acute type a aortic dissection. Circulation 2002;105:200-6.

2. Patel YD. Rupture of an aortic dissection into the pericardium. Cardiovascular Intervent Radiol 1986;9:2224.

3. John AM, Yadar S. Evaluation of blood pressure variations during the administration of intravascular contrast media in CECT Abdomen. Asian J Pharm Clin Res 2018;11:30911.

4. Adler Y, Charron P, Imazio M, et al. 2015 ESC Guidelines for the diagnosis and management of pericardial diseases: The Task Force for the Diagnosis and Management of Pericardial Diseases of the European Society of Cardiology (ESC). Endorsed by: The European Association for CardioThoracic Surgery (EACTS). Eur Heart J 2015;36:2921-64.

5. Hayashi $T$, Tsukube $T$, Yamashita $T$, et al. Impact of controlled pericardial drainage on critical cardiac tamponade with acute type A aortic dissection. Circulation. 2012;126(11 Suppl 1):S97-S101. 